\title{
Effects of Ca-enrichment on authigenic carbonate formation at cold vent sites off Costa Rica: a numerical model approach
}

\author{
D. Karaca*, C. Hensen and K. Wallmann
}

Ifm-Geomar, Wischhofstrasse 1-3, D-24148, Kiel, Germany

THE Costa Rican forearc is characterized by active fluid venting related to mud diapirism and volcanism. The geochemical compositions of fluids obtained from dewatering sites indicate that mineral precipitation or dehydration is the major driver of fluid mobilization and upward migration. The peculiar situation at the latter sites is that $\mathrm{Ca}$ concentrations in the upward migrating fluids are well above seawater levels. In turn, these Ca-enriched fluids could offer a potential reason for widespread carbonate caps on top of the mounds. Here, a reactive-transport numerical model is applied to investigate the main biogeochemical processes at cold vents off Costa Rica (Fig. 1). It is used to determine the vent- driven $\mathrm{CaCO}_{3}$ (calcium carbonate for solid phase) precipitation, quantification of methane turnover and Anaerobic Oxidation of Methane (AOM) at five different stations with varying $\mathrm{Ca}$ concentrations (Table 1). We also analysed the control parameters such as $\mathrm{Ca}$ concentrations and saturation state affecting $\mathrm{CaCO}_{3}$ precipitation rates. Fluids from two of the five sites (Culebra Fault, and Mudpie) are enriched in $\mathrm{Ca}$. In contrast, fluids at the other two sites (Mound 11 and Quepos Slide) have relatively small amounts of $\mathrm{Ca}$.

At the active-vent location of Mound 11, 98\% of the $\mathrm{CH}_{4}$ is released into the bottom waters due to the high advection rates $\left(200 \mathrm{~cm} \mathrm{y}^{-1}\right)$. The smaller $\mathrm{CH}_{4}$ turnover by $\mathrm{AOM}$ at Mound 11 also

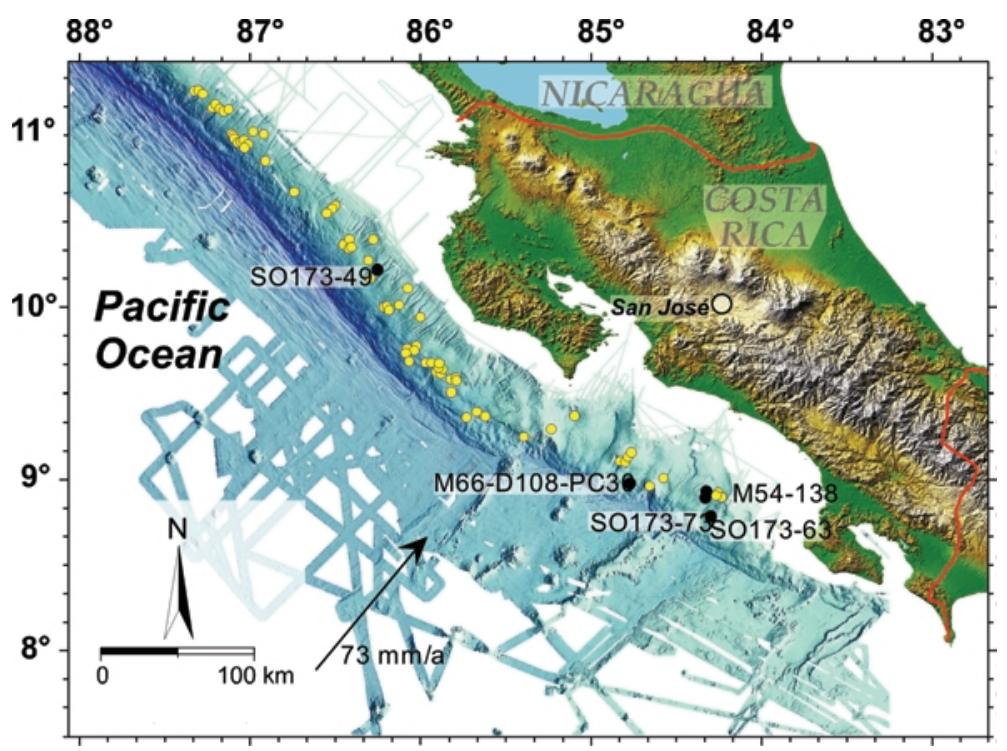

FIG. 1. Location of the study area and sampling vent sites (M54-138, SO173-73, SO173-63, M66/D78-PC30 and SO173-49) off Costa Rica.

* E-mail: dkaraca@ifm-geomar.de

DOI: $10.1180 /$ minmag.2008.072.1.325 
TABLE 1. Summary of the parameter values used as boundary values for modelling. BW indicates concentrations of dissolved species at the upper boundary of the model column, whereas BS is the concentrations of dissolved species at the bottom of the sediment column.

\begin{tabular}{|c|c|c|c|c|c|c|}
\hline & M54-138 & SO173-73 & SO173-63 & M66-PC30 & SO173-49 & \\
\hline \multicolumn{7}{|c|}{ Pore-water boundary values } \\
\hline $\mathrm{BW} \mathrm{O}_{2} / \mathrm{BS} \mathrm{O}_{2}$ & 0 & 0 & 0 & 0 & 0 & $\mathrm{~mm}$ \\
\hline $\mathrm{BW} \mathrm{NO} / \mathrm{BS} \mathrm{NO}$ & 0 & 0 & 0 & 0 & 0 & $\mathrm{~mm}$ \\
\hline $\mathrm{BW} \mathrm{Cl} / \mathrm{BS} \mathrm{Cl}$ & $551 / 210$ & $555 / 324$ & $522 / 321$ & $551 / 381$ & $550 / 291$ & $\mathrm{~mm}$ \\
\hline $\mathrm{BW} \mathrm{SO} / \mathrm{BS} \mathrm{SO}_{4}$ & $28.19 / 0$ & $28.32 / 0$ & $28.19 / 0.1$ & $26.93 / 0.37$ & $28.77 / 0$ & $\mathrm{~mm}$ \\
\hline $\mathrm{BW} \mathrm{Ca/BS} \mathrm{Ca}$ & $0 / 9.16$ & $0 / 8.08$ & $0 / 4.11$ & $0 / 31.10$ & $0 / 22.43$ & $\mathrm{~mm}$ \\
\hline $\mathrm{BW} \mathrm{CH}_{4} / \mathrm{BS} \mathrm{CH}_{4}$ & $0 / 68$ & $0 / 68$ & $0 / 68$ & $0 / 68$ & $0 / 68$ & $\mathrm{~mm}$ \\
\hline $\begin{array}{l}\mathrm{BW} \text { total } \mathrm{PO}_{4} / \\
\mathrm{BS} \text { total } \mathrm{PO}_{4}\end{array}$ & $\begin{array}{c}4.06 / 3.25 \times \\
10^{-3}\end{array}$ & $\begin{array}{c}3.45 / 3.45 \times \\
10^{-3}\end{array}$ & $\begin{array}{c}3.49 / 19.92 \times \\
10^{-3}\end{array}$ & $\begin{array}{c}1.87 / 2.87 \times \\
10^{-3}\end{array}$ & $\begin{array}{c}6.10 / 1.36 \times \\
10^{-3}\end{array}$ & $\mathrm{~mm}$ \\
\hline $\begin{array}{l}\text { BW total } \mathrm{NH}_{4} / \\
\text { BS total } \mathrm{NH}_{4}\end{array}$ & $\begin{array}{c}3.70 / 1744 \times \\
10^{-3}\end{array}$ & $\begin{array}{c}7.94 / 2905 \times \\
10^{-3}\end{array}$ & $\begin{array}{c}105.83 / 2565 \times \\
10^{-3}\end{array}$ & $\begin{array}{c}218 / 3578 \times \\
10^{-3}\end{array}$ & $0 / 6557 \times 10^{-3}$ & $\mathrm{~mm}$ \\
\hline $\mathrm{BW} \mathrm{H}_{2} \mathrm{~S} / \mathrm{BS} \mathrm{H}_{2} \mathrm{~S}$ & $0 / 0$ & $0 / 0$ & $0 / 0$ & $0 / 0$ & $0 / 0$ & $\mathrm{~mm}$ \\
\hline BW HS/BS HS & $0 / 0$ & $0 / 0$ & $0 / 0$ & $0 / 0$ & $0 / 0$ & $\mathrm{~mm}$ \\
\hline \multicolumn{7}{|c|}{ Solid species boundary values } \\
\hline $\mathrm{CaCO}_{3}$ & 4.24 & 4.24 & 4.24 & 4.24 & 4.24 & wt. $\%$ \\
\hline
\end{tabular}

causes reduced alkalinity production and as a result retards the formation of authigenic carbonates. In comparison, moderate flow rates $\left(0.1-40 \mathrm{~cm} \mathrm{y}^{-1}\right)$ at Culebra Fault, Quepos Slide and Mudpie lead to reduced $\mathrm{CH}_{4}$ output (Fig. 2). The greater efficiency of AOM and Ca fluxes here increases the $\mathrm{CaCO}_{3}$ precipitation rates, thus larger $\mathrm{Ca}$ fluxes from below induce more
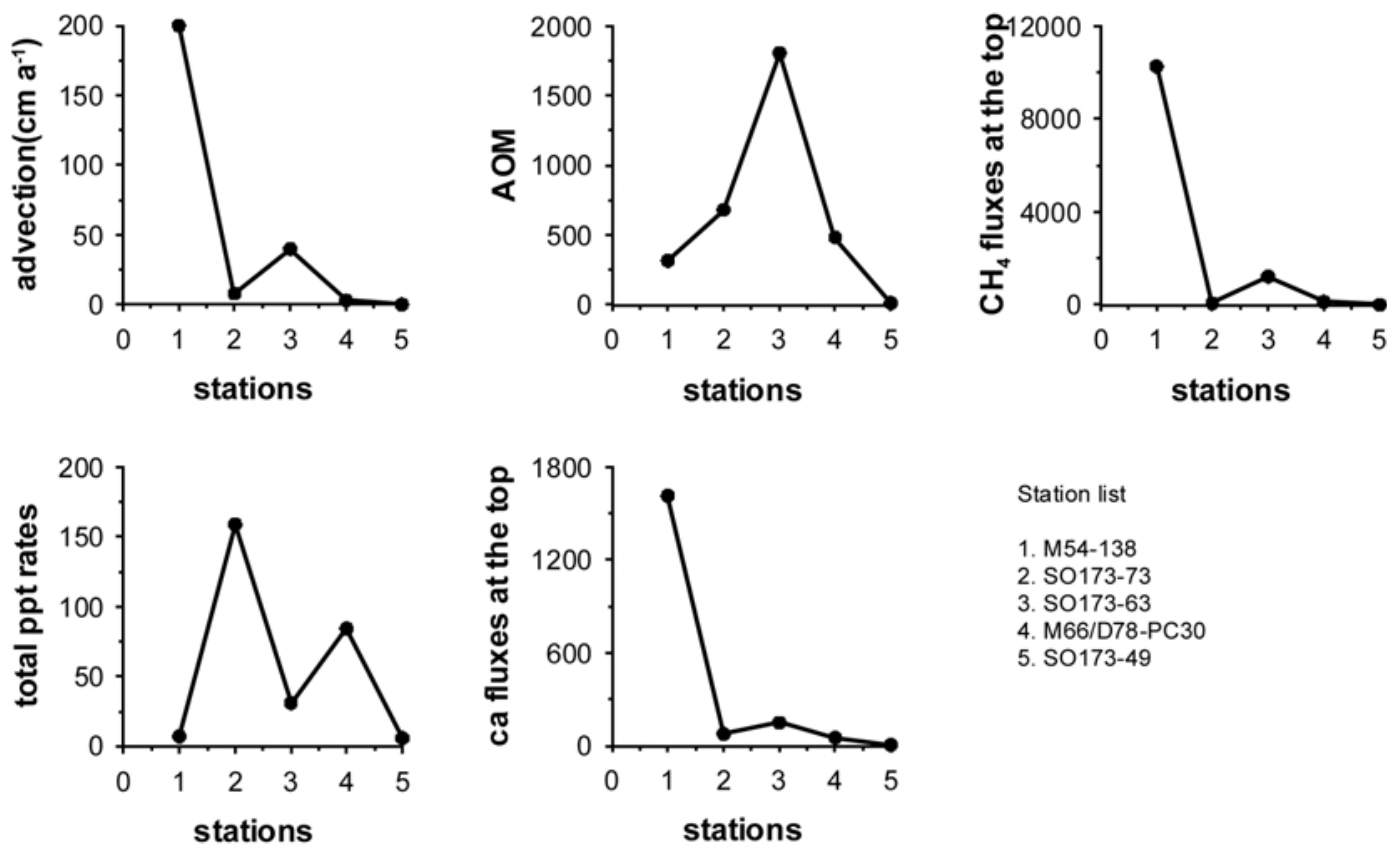

Station list

1. $M 54-138$

2. $\mathrm{SO} 173-73$

3. SO173-63

4. M66/D78-PC30

5. SO173-49

FIG. 2. Summary of advection $\left(\mathrm{cm} \mathrm{y}^{-1}\right)$, turnover and $\mathrm{CaCO}_{3}$ precipitation rates $\left(\mu \mathrm{mol} \mathrm{cm}^{-2} \mathrm{y}^{-1}\right)$ at simulated stations. 


\section{Calcite ppt}

\section{Aragonite ppt}

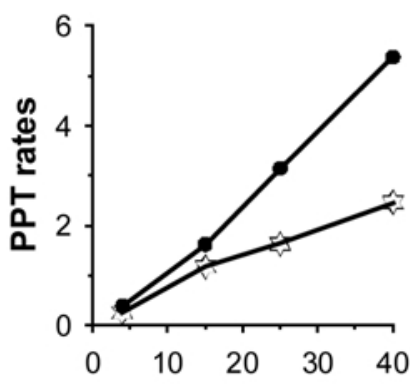

$\left[\mathrm{Ca}^{2+}\right]$ in fluid $(\mathrm{mM})$ $u=200 \mathrm{~cm} \mathrm{a}^{-1}$

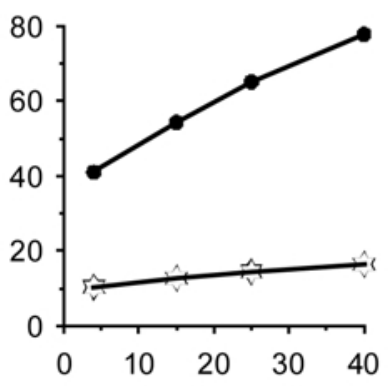

$\left[\mathrm{Ca}^{2+}\right]$ in fluid $(\mathrm{mM})$

$$
\mathrm{u}=10 \mathrm{~cm} \mathrm{a}{ }^{-1}
$$

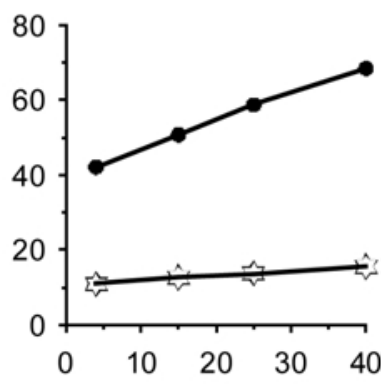

$\left[\mathrm{Ca}^{2+}\right]$ in fluid $(\mathrm{mM})$

$$
\mathrm{u}=1 \mathrm{~cm} \mathrm{a}^{-1}
$$

FIG. 3. Diagrams showing how the precipitation rates of calcite and aragonite $\left(\mu \mathrm{mol} \mathrm{cm}{ }^{-2} \mathrm{y}^{-1}\right)$ respond to variations of $\mathrm{Ca}$ concentrations in the ascending fluids.

precipitation of $\mathrm{CaCO}_{3}$. Simulations with varying fluid-flow rate and increased $\mathrm{Ca}$ concentrations in the ascending fluid demonstrate that the impact of Ca-enrichment from fluid venting on carbonate precipitation rate is significant (Fig. 3). This effect is more pronounced at smaller advection rates. The saturation state of upward migrating fluids is a sensitive parameter affecting the $\mathrm{CaCO}_{3}$ precipitation in surface sediments, particularly at large advection rates. 